\title{
CHROMOSOMES, KARYOTYPE ANALYSIS, CHROMOSOME REARRANGEMENTS IN FUNGI
}

\author{
L. HORNOK \\ Agricultural Biotechnology Center and University of Agricultural Sciences, Gödöllô, Hungary
}

In this review the organization of fungal chromosomes and the methods used for karyotype analysis are briefly summarized. The role of chromosome rearrangement, supernumerary chromosomes and repeated DNA sequences in the genetic change of fungi is evaluated

\section{Fungal chromosomes}

The genome organization of fungi is typical of eukaryotic systems. The chromosomes are composed of DNA, as well as histone and non-histone proteins. The size of the haploid nuclear genome of fungi ranges between 15-60 Mb (megabase pairs), the number of chromosomes varies from three to a few dozens depending on species. The chromosomes are organized into nucleosomes and contain a localized centromerekinetochore complex (which is the nucleating site for the spindle microtubules), as well as telomeres (that maintain the integrity of the chromosomes and help in directing chromosome pairings). A typical spindle mechanism is the driving force of mitosis divided into a normal prophase, metaphase and a non-synchronous anaphase-telophase taking together $6 \mathrm{~min}$. under optimal conditions. The meiotic process - like in other eukaryotes - includes DNA replication (pre-meiotic S phase), as well as one reductional (meiosis I) and one equational (meiosis II) division. In the majority of fungi the meiotic S phase precedes karyogamy.

\section{Karyotype analysis}

Chromosome numbers and sizes (the karyotype) can be determined by light and electron microscopy as well as by pulsed-field gel electrophoresis. The latter technique used for the first time in 1984 to separate yeast chromosome-sized DNAs [1] - raised a

LÁSZLÓ HORNOK

Agricultural Biotechnology Center

P.O. Box 411, H-2101 Gödöllô, Hungary 
revolution in fungal genetics. Since then, numerous electrophoretic karyotypes for a wide range of fungi have been published. Hungarian workers provided the first comparative karyotype analysis for selected species of several economically important genera, like Fusarium, Mucor, Phaffia and Trichoderma [2-5]. Cloned DNA fragments may be assigned to electrophoretically separated chromosomes by Southern hybridization, therefore molecular karyotyping allows direct gene mapping and identification of linkage relationships. Chromosome-specific libraries may also be prepared after separation of chromosome-sized DNAs. Chromosome-specific probes may be used to recognize homologous chromosomes within polymorphic chromosome patterns. Karyotype analysis may provide additional information for the appropriate taxonomic affiliation of controversial species.

\section{Chromosome polymorphisms}

Genomic rearrangements play an important role in the evolution of fungi [6]. Such rearrangements may result in chromosome polymorphisms found to be accompanied by phenotype differences including morphology [7], antibiotic production [8] or host range [9].

Karyotype analysis revealed high levels of variation in chromosome sizes and/or numbers within most species of fungi. According to a persuasive hypothesis proposed by Kistler and Miao [10] the extent of intraspecific karyotype polymorphism is inversely correlated to the frequency of meiosis. Asexual fungi have more extensive polymorphisms than sexual ones, which frequently undergo meiosis, an efficient process selecting against detectable chromosome aberrations. Both meiotic and mitotic processes may lead to chromosome polymorphisms and genomic stress can also account for karyotype variability. Repeated DNA sequences may also generate chromosome polymorphisms (see below).

For asexual plant pathogenic fungi, variation in chromosome sizes and numbers is an efficient non-Mendelian mechanism of genetic change resulting in the emergence of virulent races or pathotypes.

\section{Supernumerary chromosomes}

The occurrence of 'extra' chromosomes is very common in fungi. Various terms (B chromosomes, dispensable chromosomes, mini-chromosomes and supernumerary chromosomes) have been used for naming these structures. According to a recent review of this topic [11] the term 'supernumerary chromosomes' was suggested as a preferred name. A chromosome could be qualified as a supernumerary one, if it is not present in one or other viable individual of a given species. In sexual fungi the supernumerary chromosomes are inherited by non-Mendelian transmission, their absence is non-lethal. In spite of this seemingly dispensable nature of these elements there are examples for the long-term mitotic stability of supernumerary chromosomes $[12,13]$. 
The movement of supernumerary chromosomes in pulse-field gels, their structural stability during mitosis and their positive hybridization to telomeric DNA probes indicate strongly that these elements contain centromeres and telomeres. Information on DNA composition of these chromosomes is limited to a few species. A mixture of repeated as well as low- and single-copy DNA sequences have been identified in supernumerary chromosomes of Colletotrichum gloeosporioides and Nectria haematococca [14, 15]. In our experiments mini-chromosomes in Fusarium sporotrichioides (some of them were undoubtedly supernumerary ones) were found to constitute a mosaic composed of repeated DNAs dispersed throughout the genome and unique sequences. These chromosomes were most probably formed by major genomic rearrangements and were preserved during the evolution of the species. The mosaic structure was identified in all genetically isolated strains of the fungus indicating that the mini-chromosomes - although lacking any coding region - could be useful components of the genome [12].

The biological function of supernumerary chromosomes is cryptic except for two notable cases. The $1.6 \mathrm{Mb}$ dispensable chromosome of $N$. haematococca, a facultative plant pathogen carries a gene (PDA1-1) encoding the enzyme, pisatin demethylase, which catalyses the detoxification of pisatin, an antifungal phytoalexin of pea. Strains that retained this supernumerary chromosome show a high level of pisatin detoxification activity and are highly virulent on Pisum sativum [16]. A supernumerary chromosome of Cochliobolus carbonum race 1 isolates carries genes responsible for the synthesis of HCtoxin, a host specific cyclic tetrapeptide which causes disease symptoms on maize [17]; strains lacking this chromosome (and the genetic determinants of HC-toxin production) are avirulent on maize.

\section{Repeated DNA sequences}

The fungal genome harbors interspersed repetitive elements, that may be mobile or stably integrated elements. The mobile elements are classified as (i) transposons, which transpose through DNA copies and have an open reading frame encoding transposase activity, (ii) retrotransposons, which duplicate via RNA intermediates and have retroviruslike structures and (iii) retroposons, which also use RNA intermediates during replication but lack the structural features of retroviruses. The fungal retroposons are further subgrouped into either as short interspersed nuclear elements (SINEs) or long interspersed nuclear elements (LINEs). The first fungal transposon, Fotl discovered in the plant pathogen Fusarium oxysporum was found to share structural similarities with the class of Tcl-like elements [18]. Of the retrotransposons, Ty4 present in Saccharomyces cerevisiae was the first element subjected to complete molecular analysis [19]. CfT-1 isolated from Cladosporium fulvum was a pioneer example of the LTR-type (long terminal repeat) retrotransposons in filamentous fungi [20]. A short interspersed nuclear element (MgSINE) was isolated from the rice blast fungus, Magnaporthe grisea [21], whereas LINElike elements were for the first time detected in Neurospora crassa [22]. Numerous other examples of the mobile repetitive sequences have subsequently been reported and some of these sequences proved to be functional transposable elements. 
Of the stably integrated (non-rDNA) repeated DNA sequences in fungi one class is characterized as telomere-associated elements, present on all or almost all chromosomes. Such telomeric sequences, like Ca7 [23] or the Rel-2 element [24] of Candida albicans are considered essential for proper chromosome maintenance. The second class of the stably integrated non-rDNA repeats is represented by the CARE-1 element [25] and the RPS sequences cloned from $C$. albicans [26]. These elements are not present on all resolvable chromosomes. CARE-1 was identified as a non-telomeric, probably nonessential sequence with a copy number ranging between two and twelve per haploid genome, depending on strain, whereas the RPS 1 element, represented by some 80 copies was found to be present on all but one chromosome of C. albicans.

In our laboratory a moderately repetitive DNA element has been cloned from Fusarium poae a strictly asexual fungus, which is a secondary invader of small-grain cereals. The element, named ZIT1 selectively hybridized to the polymorphic, 1.0-3.7 Mb chromosomal region of the fungus, but no hybridization signal was observed on any of the large chromosomes. ZIT1 shares only a moderate level of similarity to gag genes of fungal retrotransposons, which was mainly due to a small cysteine-reach motif, known as zinc-finger DNA binding domain. No significant homology was found with any other published nucleotide sequence. The novelty of this element is that its distribution is restricted to the polymorphic chromosome region of $F$. poae. ZIT1 could be a remnant of a formerly active foreign DNA that had been eliminated from the large chromosomes by a defensive mechanism suitable to prevent the disruption of genes encoding vital functions [27].

Repetitive DNA sequences are regarded to be important determinants of genetic variability in clonal eukaryotic organisms, like most hyphomycete fungi in which recombination through sexual or parasexual cycles is rare or, in fact absent. Transposable elements have been shown to cause chromosome rearrangement and various aberrations like deletions, inversions and translocations. Repeated DNA sequences, even if they are unable to transpose may generate DNA rearrangements through recombination among their homologous sequences scattered throughout the genome.

Acknowledgement. The original research cited in this review has been supported by a Hungarian Scientific Research Grant (OTKA T-019315).

\section{REFERENCES*}

1. Schwartz,D.C., Cantor,C.R.: Separation of yeast chromosome-sized DNAs by pulsed field gradient gel electrophoresis. Cell 37, 67 (1984).

2. Fekete,C., Nagy,R., Debets,A.J.M., Hornok,L.: Electrophoretic karyotypes and gene mapping in eight species of the Fusarium sections Arthrosporiella and Sporotrichiella. Curr Genet 24, 500 (1993).

3. Fekete,C., Weszely,T., Hornok,L.: Assignment of a PCR-amplified chitinase sequence cloned from Trichoderma hamatum to resolved chromosomes of potential biocontrol species of Trichoderma. FEMS Microbiol Lett 145, 385 (1996).

4. Nagy,Á., Vágvölgyi,Cs., Balla,E., Ferenczy,L.: Electrophoretic karyotype of Mucor circinelloides. Curr Genet 26, 45 (1994). 
5. Nagy,Á., Garamszegi,N., Vágvölgyi,Cs., Ferenczy,L.: Electrophoretic karyotypes of Phaffia rhodozyma strains. FEMS Microbiol Lett 123, 315 (1994).

6. Smith,A.W., Collis,K., Ramsden,M., Fox,H.M., Peberdy,J.F.: Chromosome rearrangement in improved cephalosporin C-producing strains of Acremonium chrysogenum. Curr Genet 19, 235 (1991).

7. Suzuki,T., Miyamae,Y., Ishida,L.: Variation of colony morphology and chromosomal rearrangement in Candida tropicalis pK233. J Gen Microbiol 137, 161 (1991).

8. Walz,M., Kück,U.: Polymorphic karyotypes in related Acremonium strains. Curr Genet 19, 73 (1991).

9. Cooley,R.N., Caten,C.E.: Variation in electrophoretic karyotype between strains of Septoria nodorum. Mol Gen Genet 228, 17 (1991).

10. Kistler,H.C., Miao,V.P.W.: Now modes of genetic change in filamentous fungi. Annu Rev Phytopathol 30 , 131 (1992).

11. Covert,S.F.: Supernumerary chromosomes of filamentous fungi. Curr Genet 33, 311 (1998).

12. Nagy,R., Táborhegyi,E., Wittner,A., Hornok,L.: Mini-chromosomes in Fusarium sporotrichioides are mosaics of dispersed repeats and unique sequences. Microbiology 141, 713 (1995).

13. VanEtten,H.D., Jorgensen,S., Enkerli,J., Covert,S.F.: Inducing the loss of dispensable chromosomes in Nectria haematococca during vegetative growth. Curr Genet 33, 299 (1998).

14. Kim,H.G., Meinhardt,L.W., Benny,U., Kistler,H.C.: Nrs1, a repetitive element linked to pisatin demethylase genes on a dispensable chromosome of Nectria haematococca. Mol Plant Microbe Interact 8 (1995).

15. Masel,A.M., He,C., Poplawski,A.M., Irwin,J.A.G., Manners,J.M.: Molecular evidence for chromosome transfer between biotypes of Colletotrichum gloeosporioides. Mol Plant-Microbe Interact 9, 339 (1996).

16. Miao,V.P.W., Matthews,D.E., VanEtten,H.D.: Identification and chromosomal locations of a family of cytochrome P-450 genes for pisatin detoxification in the fungus Nectria haematococca. Mol Gen Genet 226, 214 (1991).

17. Ahn,J.-H., Walton,J.D.: Chromosomal organization of TOX2, a complex locus controlling host-selective toxin biosynthesis in Cochliobolus carbonum. The Plant Cell 8, 887 (1996).

18. Daboussi,M.-J., Langin,T., Brygoo,Y.: Fot1, a new family of fungal transposable elements. Mol Gen Genet 232, 12 (1992)

19. Stucka,R., Schwarzlose,C., Lochmüller,H., Hacker,U., Feldmann,H.: Molecular analysis of the yeast Ty4 element: homology with Tyl, copia and plant retrotransposons. Gene 122, 119 (1992).

20. McHale,M.T., Roberts,I.N., Noble,S.M., Beaumont,C., Whitehead,M.P., Seth, D., Oliver,R.P.: CfT-1: an LTR-retrotransposon in Cladosporium fulvum, a fungal pathogen of tomato. Mol Gen Genet 223, 337 (1992).

21. Kachroo,P., Leong,S.A., Chattoo,B.B.: Mg-SINE: A short interspersed nuclear element from the rice blast fungus, Magnaporthe grisea. Proc Natl Acad Sci USA 92, 11125 (1995).

22. Kinsey,J.A., Helber,J.: Isolation of a transposable element from Neurospora crassa. Proc Natl Acad Sci USA 86, 1929 (1989)

23. Sadhu,C., McEachern,M.J., Rustchenko-Bulgac,E.P., Schmid,J., Soll,D.R., Hicks, J.B.: Telomeric and dispersed repeat sequences in Candida yeasts and their use in strain identification. J Bacteriol 173, 842 (1991).

24. Thrash-Bingham,C., Gorman,J.A.: Identification, characterization and sequence of Candida albicans repetitive DNAs Rel-1 and Rel-2. Curr Genet 23, 455 (1993).

25. Lasker,B.A., Page,L.S., Lott,T.J., Kobayashi,G.S., Medoff,G.: Characterization of CARE-1: Candida albicans repetitive element-1. Gene 102, 45 (1991).

26. Chindamporn,A., Nakagawa,Y., Homma,M., Chibana,H., Doi,M., Tanaka,K.: Analysis of the chromosomal localization of the repetitive sequences (RPSs) in Candida albicans. Microbiology 141, 469 (1995).

27. Fekete,C., Hornok,L: A repetitive DNA sequence associated with karyotype variability in Fusarium poae. Acta Phytopath Entomol Hung 32, 29 (1997). 
*Due to spatial limitations of this review the coverage of literature is only partial. Non-intentional omissions could not be avoided. 